This item was submitted to Loughborough's Research Repository by the author.

Items in Figshare are protected by copyright, with all rights reserved, unless otherwise indicated.

\title{
Optimization of catalyst structure for asymmetric propargylation of aldehydes with allenyltrichlorosilane
}

PLEASE CITE THE PUBLISHED VERSION

https://doi.org/10.1002/adsc.202000936

PUBLISHER

Wiley

VERSION

AM (Accepted Manuscript)

\section{PUBLISHER STATEMENT}

This is the peer reviewed version of the following article: VAGANOV, V.Y. ... et al, 2020. Optimization of catalyst structure for asymmetric propargylation of aldehydes with allenyltrichlorosilane. Advanced Synthesis \& Catalysis, 362 (23), pp.5467-5474, which has been published in final form at https://doi.org/10.1002/adsc.202000936. This article may be used for non-commercial purposes in accordance with Wiley Terms and Conditions for Use of Self-Archived Versions.

\section{LICENCE}

CC BY-NC-ND 4.0

\section{REPOSITORY RECORD}

Vaganov, Vladimir, Yasuaki Fukazawa, Nikolay S. Kondratyev, Sergei A. Shipilovskikh, Steven Wheeler, Aleksandr Rubtsov, and Andrei Malkov. 2020. "Optimization of Catalyst Structure for Asymmetric Propargylation of Aldehydes with Allenyltrichlorosilane". Loughborough University. https://hdl.handle.net/2134/13007078.v1. 


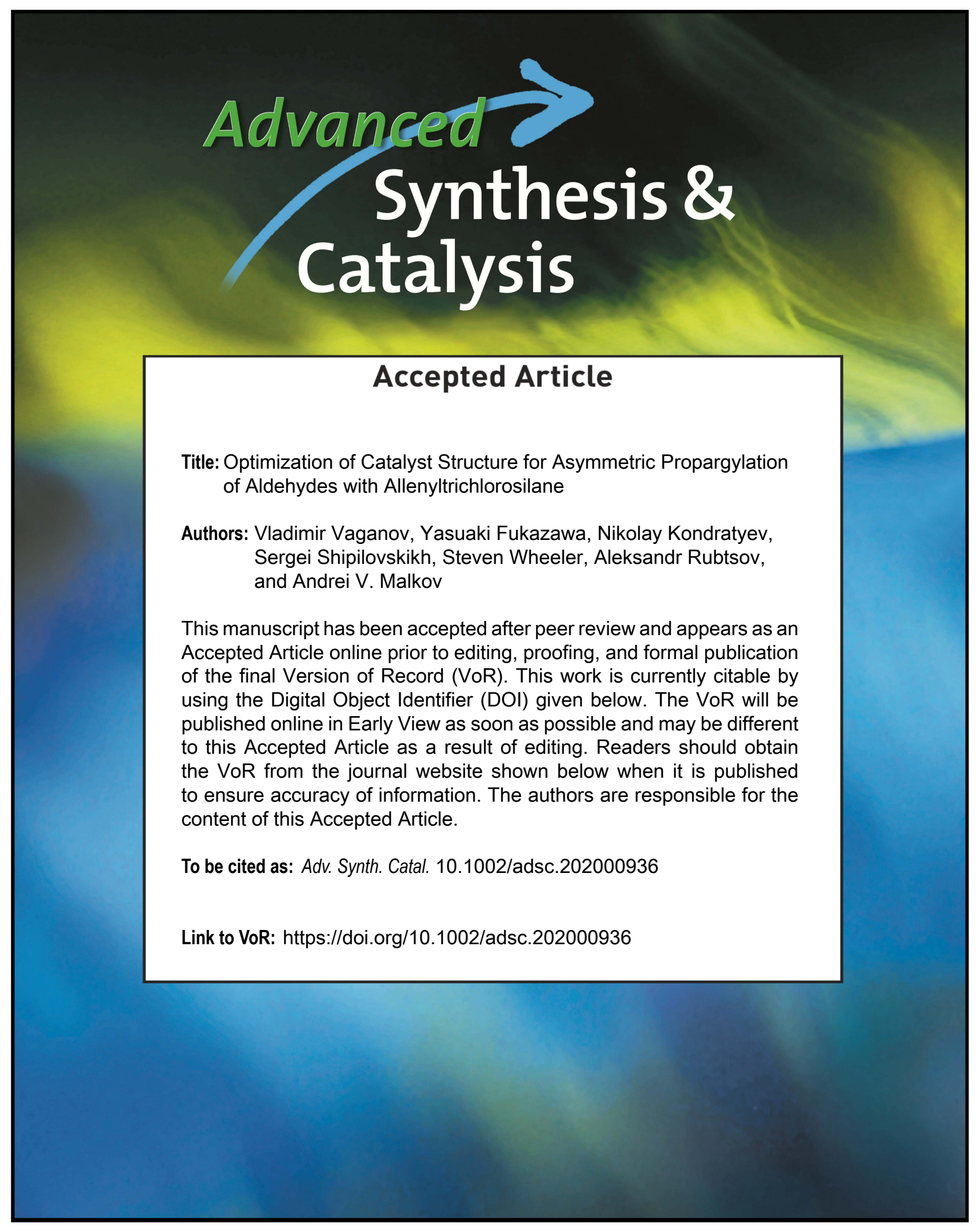




\title{
Optimization of Catalyst Structure for Asymmetric Propargylation of Aldehydes with Allenyltrichlorosilane
}

\author{
Vladimir Yu. Vaganov, ${ }^{a}$ Yasuaki Fukazawa, ${ }^{b}$ Nikolay S. Kondratyev, ${ }^{b}$ Sergei A. \\ Shipilovskikh, ${ }^{\mathrm{ab}}$ Steven E. Wheeler, ${ }^{\mathrm{c}}$ Aleksandr E. Rubtsov, ${ }^{\mathrm{a} *}$ and Andrei V. Malkov ${ }^{\mathrm{b} *}$ \\ a Department of Chemistry, Perm State University, Bukireva 15, Perm 614990, Russia. E-mail:rubtsov@psu.ru \\ b Department of Chemistry, Loughborough University, Loughborough, LE113TU, UK. E-mail: A.Malkov@lboro.ac.uk \\ c Department of Chemistry, University of Georgia, Athens, GA, USA.
}

Received: ((will be filled in by the editorial staff))

Supporting information for this article is available on the WWW under http://dx.doi.org/10.1002/adsc.201\#\#\#\#\#\#.

\begin{abstract}
The design of catalysts for asymmetric propargylations remains a challenging task, with only a handful of methods providing access to enantioenriched homopropargylic alcohols. In this work, guided by previously reported computational predictions, a set of atropisomeric bipyridine $N, N^{\prime}$-dioxides was tested as Lewis base catalysts for the asymmetric propargylation of aldehydes with trichloroallenylsilane. The catalysts are easily prepared in four simple steps starting from readily available methyl ketones. Aryl-substituted derivatives proved to be highly active and showed a high level of enantiocontrol even at $1 \mathrm{~mol} \%$ loading.
\end{abstract}

The reaction scope includes a wide range of aromatic, heteroaromatic, and unsaturated aldehydes. New computations confirm that the key stereodetermining transition state structures for the synthesized catalysts are similar to those previously reported for the model structure.

Keywords: asymmetric catalysis, enantioselectivity, propargylation, Lewis bases, atropisomerism

\section{Introduction}

The asymmetric nucleophilic propargylation of aldehydes is a convenient method for the formation of $\mathrm{C}-\mathrm{C}$ bonds that introduces an unsaturated fragment into the molecule. While representing a complementary methodology to the widely used allylation reaction, the propargylation reaction stays in the shadow of its more established counterpart despite the fact that the resultant homopropargylic alcohols serve as versatile synthetic building blocks. ${ }^{[1]}$ Typically, the protocols involve addition of preformed or made in situ organometallic propargylating reagents to carbonyl compounds in the presence of a catalyst. Recently, alternative methods emerged employing 1,3-enynes as allenylmetal equivalents in transition metal mediated asymmetric propargylation. ${ }^{[2]} \quad$ Transition metal-free methodologies for asymmetric propargylations of carbonyl compounds mostly revolve around organoboron and organosilicon reagents, the former being more actively exploited. ${ }^{[3]}$ Thus, boron reagents have been successfully employed in the catalytic asymmetric propargylation of both aldehydes ${ }^{[4]}$ and ketones. ${ }^{[5]}$ Asymmetric synthesis employing the respective silicon reagents, alongside their tin counterparts, in the past was mostly focused on the use of enantioenriched chiral derivatives where a non-chiral catalyst was used to facilitate the addition $^{[6]}$ with a few examples of chiral catalysis. ${ }^{[}$ With the advent of asymmetric Lewis base catalysis, ${ }^{[8]}$ the propargylation of aldehydes 1 using allenyltrichlorosilane $2^{[9]}$ started to attract the attention of researchers (Scheme 1); however, it proved considerably more challenging than the related allylation, which is reflected in just a handful of published reports on this transformation.

For instance, Nakajima's biquinoline $N, N$ '-dioxide 4 (20 mol\% loading) afforded homopropargylic alcohols 3 with modest enantioselectivity $(\leq 52 \%$ ee),${ }^{[10]}$ in contrast to the results attained in the respective allylation $\left(\leq 92 \%\right.$ ee).$^{[11]}$ On the other hand, highly enantioselective formation of $\mathbf{3}(\leq 96 \%$ ee) has been reported for the helical pyridine $N$-oxide $\mathbf{5}$ as catalyst (at $10 \quad \mathrm{~mol} \%$ loading). ${ }^{[12]}$ Several computational studies were carried out to address the apparent disparity in the enantioselectivities seen in the allylation and propargylation of aldehydes catalyzed by bipyridine $N$-oxides or $N, N$ '-dioxides and also to gain a deeper insight into the mechanism of stereodifferentiation in the asymmetric propargylation. ${ }^{[13]}$ For example, the high enantioselectivity attained with $\mathbf{5}$ resulted, in part, from the through-space electrostatic interactions of the formyl $\mathrm{C}-\mathrm{H}$ with the $\mathrm{Cl}$ ligands on $\mathrm{Si}$, rather than noncovalent aryl-aryl interactions between the aromatic aldehyde and the catalyst. ${ }^{[13 \mathrm{~b}]}$ Wheeler and co-workers subsequently developed the computational toolkit AARON (Automated 
Alkylation Reaction Optimizer for $N$-oxides), which enabled virtual screening of various bipyridine $N, N^{\prime}$ dioxides scaffolds for designing highly selective catalysts for the asymmetric propargylation of aldehydes.

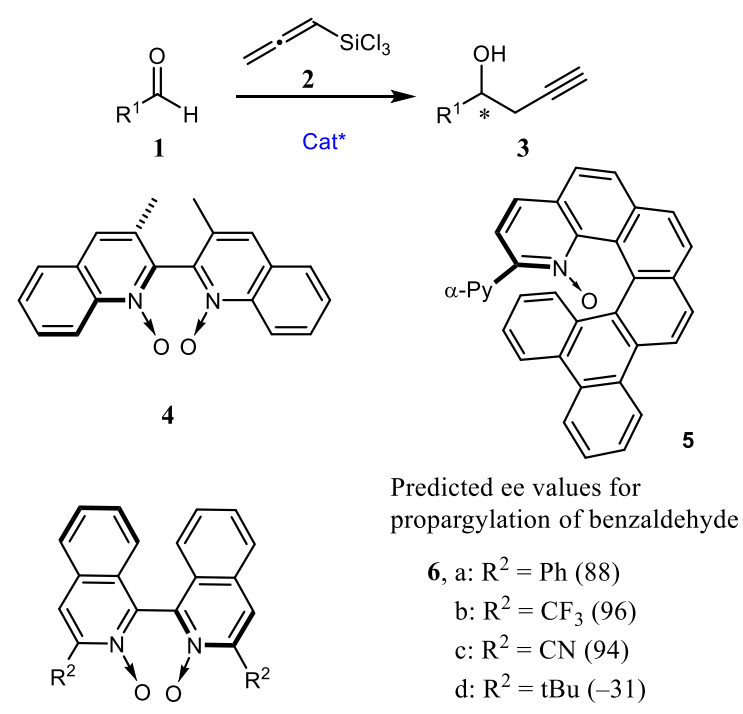

Scheme 1. Asymmetric propargylation of aldehydes catalyzed by pyridine $N$-oxides.

Axially chiral $N, N$ '-dioxides 6 were identified as promising candidates (Scheme 1) where certain derivatives were predicted to show high enantioselectivity. ${ }^{[14]}$ However, it is important to mention that these computational predictions only considered selectivity and did not take into account catalytic activity, adding further need for experimental verification of the proposed structures. Herein, we present a practical assessment of close analogues of the theoretically proposed catalyst structures in asymmetric propargylation of aldehydes with allenyltrichlorosilane, leading to the identification of a novel, highly efficient catalytic system.

\section{Results and Discussion}

Recently, we developed a short and convenient method for the stereoselective synthesis of terpenederived atropisomeric bipyridine $N, N^{\prime}$-dioxides 7, where substituents in the pyridine ring can be varied through the choice of the starting ketone 8 (Scheme 2). ${ }^{[15]}$ Where the desired substituent cannot be introduced through the Kroenke annulation $(\mathbf{9} \rightarrow \mathbf{1 1})$, chemical modification of the available groups in pyridine $\mathbf{1 1}$ may come to the rescue (e.g. 2-furyl derivative 11i was transformed into nitrile 11c, see SI for details). Notably, compound $7 \mathrm{e}$ exhibited excellent enantiocontrol in the crotylation of aldehydes; ${ }^{[16]}$ therefore, it was reasonable to investigate $N, N^{\prime}$-dioxides 7 as catalysts in the asymmetric propargylation. While 7 is different from the original structures explored computationally (6), they offer the practical advantage of being synthesized in pure diastereomeric forms. Furthermore, as shown below computationally, the transition state (TS) structures for 7 are nearly identical to the corresponding substituted form of $\mathbf{6}$.

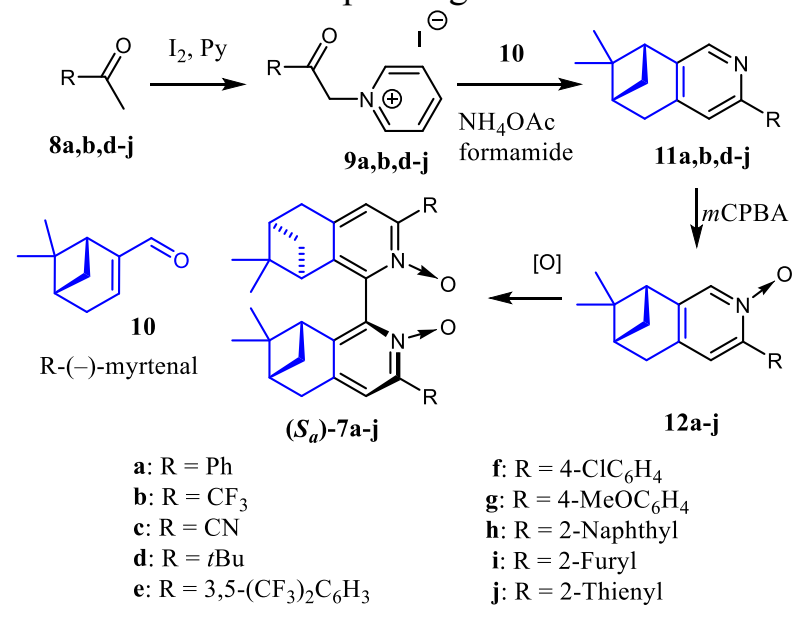

Scheme 2. Synthesis of atropisomeric bipyridine N,N'dioxides 7.

A set of compounds employed in this work, including derivatives with the substitution pattern identified as optimal in the theoretical studies by Wheeler, ${ }^{[14 b]}$ is presented in Scheme 2. Catalyst performance was assessed in a model addition of allenylsilane 2 to benzaldehyde using $10 \mathrm{~mol} \%$ catalysts loading at $-60^{\circ} \mathrm{C}$ for $18 \mathrm{~h}$. The results are presented in Table 1.

Table 1. Catalyst screening in addition trichloroallenylsilane $\mathbf{2}$ to benzaldehyde $\mathbf{1 a} .^{a}$

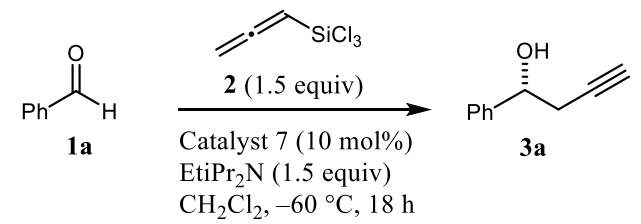

\begin{tabular}{llll}
\hline entry & catalyst 7, $\mathrm{R}$ & $\begin{array}{l}\text { conversion, } \\
\mathbf{\%}^{\mathrm{b}}\end{array}$ & $\begin{array}{l}\mathrm{ee}, \\
(\mathbf{\%})^{\mathrm{c}}\end{array}$ \\
\hline 1 & $\mathbf{7 a},\left(S_{\mathrm{a}}\right)-\mathrm{Ph}$ & 99 & 96 \\
2 & $\mathbf{7 a},\left(R_{\mathrm{a}}\right)-\mathrm{Ph}$ & 99 & $96^{\mathrm{d}}$ \\
3 & $\mathbf{7 b}, \mathrm{CF}_{3}$ & trace & n.d. \\
4 & $\mathbf{7 c}, \mathrm{CN}$ & $\leq 5$ & 69 \\
5 & $\mathbf{7 d}, t \mathrm{Bu}$ & $\leq 5$ & 50 \\
6 & $\mathbf{7 e}, 3,5-\left(\mathrm{CF}_{3}\right)_{2} \mathrm{C}_{6} \mathrm{H}_{3}$ & 99 & 93 \\
7 & $\mathbf{7 f}, 4-\mathrm{ClC}_{6} \mathrm{H}_{4}$ & 99 & 97 \\
8 & $\mathbf{7 g}, 4-\mathrm{MeOC}_{6} \mathrm{H}_{4}$ & 70 & 93 \\
9 & $\mathbf{7 h}, 2-\mathrm{Naphthyl}$ & 99 & 95 \\
10 & $\mathbf{7 i}, 2-$ Furyl & 60 & 60 \\
11 & $\mathbf{7 j}, 2-$ Thienyl & 99 & 76 \\
\hline
\end{tabular}

a) The reactions were carried out on a $0.5 \mathrm{mmol}$ scale in $\mathrm{CH}_{2} \mathrm{Cl}_{2}$ at $-60{ }^{\circ} \mathrm{C}$ under argon with $10 \mathrm{~mol} \%$ of catalyst 7 for 18 hours. ${ }^{\text {b) }}$ Conversion was calculated from GC data. ${ }^{c)}$ Determined by GC on a chiral column. ${ }^{\mathrm{d})}$ Alcohol 3a was $(S)$-configured.

Catalyst $\left(S_{\mathrm{a}}\right)-\mathbf{7 a}$, with phenyl substituents, furnished alcohol 3a in $96 \%$ ee, providing a good 
match with the theoretically predicted $88 \%$ ee for $\mathbf{6 a}$ (entry 1). ${ }^{[14 \mathrm{~b}]}$ Its atropisomer $\left(R_{\mathrm{a}}\right)-\mathbf{7 a}$, behaved in the same way furnishing alcohol $\mathbf{3 a}$ of the opposite enantiomeric series (entry 2). Analogues of other catalysts that were predicted to show high levels of enantioselectivity proved less successful. For instance, 7c, with $\mathrm{CN}$ substituents, exhibited low reactivity giving 3a in poor conversion, though with reasonable $69 \%$ ee (entry 4), whereas $7 \mathbf{b}\left(\mathrm{R}=\mathrm{CF}_{3}\right)$ proved completely inactive (entry 3 ). Catalyst $\mathbf{7 d}$, with bulky $t \mathrm{Bu}$ substituents, also reacted sluggishly and resulted in modest $50 \%$ ee (entry 5). The success of $7 \mathbf{a}$ prompted us to investigate other aromatic substituents (7e-7j). All the $N, N$ '-dioxides containing aryl groups in the $\alpha$-position to the $N$-oxide proved to be active catalysts with high enantioselectivities (entries 6-9), except for the heteroaromatic substituents where enantiomeric excess dropped somewhat (entries 10, 11).

In the related allylation, solvent was shown to exert an essential influence on the enantioselectivity of the reaction by altering the operative reaction mechanism. ${ }^{[17]}$ Therefore, in the next stage, the effect of solvent on propargylations was investigated. Representative examples are collected in Table 2 (for the full set of tests, see Tables S1-S3 in Supporting Information).

Table 2. Effect of solvent in addition of trichloroallenylsilane $\mathbf{2}$ to benzaldehyde $\mathbf{1 a} .^{\text {a }}$

\begin{tabular}{lllll}
\hline entry & catalyst 7, $\mathrm{R}$ & solvent & $\begin{array}{l}\text { convers } \\
\text { ion, } \% \text { b }\end{array}$ & $\begin{array}{l}\text { ee, } \\
(\%)\end{array}$ \\
\hline 1 & $\mathbf{7 a}, \mathrm{Ph}$ & DCM & 99 & 96 \\
2 & $\mathbf{7 a}, \mathrm{Ph}$ & EtCN & $\leq 5$ & 76 \\
3 & $\mathbf{7 e}, 3,5-\left(\mathrm{CF}_{3}\right)_{2} \mathrm{C}_{6} \mathrm{H}_{3}$ & DCM & 99 & 93 \\
4 & $\mathbf{7 e}, 3,5-\left(\mathrm{CF}_{3}\right)_{2} \mathrm{C}_{6} \mathrm{H}_{3}$ & EtCN & 14 & 93 \\
5 & $\mathbf{7 g}, 4-\mathrm{MeOC}_{6} \mathrm{H}_{4}$ & DCM & 70 & 93 \\
6 & $\mathbf{7 g}, 4-\mathrm{MeOC}_{6} \mathrm{H}_{4}$ & EtCN & 99 & 72 \\
7 & $\mathbf{7 g}, 4-\mathrm{MeOC}_{6} \mathrm{H}_{4}$ & THF & 85 & 37 \\
8 & $\mathbf{7 g}, 4-\mathrm{MeOC}_{6} \mathrm{H}_{4}$ & $\mathrm{CHCl}$ & $\leq 5$ & 80 \\
9 & $\mathbf{7 g}, 4-\mathrm{MeOC}_{6} \mathrm{H}_{4}$ & EtOAc & 43 & 74 \\
10 & $\mathbf{7 g}, 4-\mathrm{MeOC}_{6} \mathrm{H}_{4}$ & Ether & 7 & 40 \\
11 & $\mathbf{7 g}, 4-\mathrm{MeOC}_{6} \mathrm{H}_{4}$ & Toluene & 9 & 40 \\
\hline
\end{tabular}

a) The reactions were carried out on a $0.5 \mathrm{mmol}$ scale at $60{ }^{\circ} \mathrm{C}$ under argon with $10 \mathrm{~mol} \%$ of catalyst 7 for 18 hours. b) Conversion was calculated from GC data. ${ }^{\text {c) Determined }}$ by GC on a chiral column.

Dichloromethane appears to be the optimal solvent, at least for the addition to benzaldehyde (entries 1, 3, 5). In propionitrile (entries 2, 4, 6), which served well in the related crotylation, ${ }^{[16 a]}$ the results did not show any defined trend; however, enantioselectivity was generally lower than in dichloromethane. A number of other solvents were tested with catalyst $\mathbf{7 g}$ and were clearly inferior to dichloromethane in terms of both reactivity and enantioselectivity (entries 7-11).

In the initial experiments, catalyst loading was kept at $10 \mathrm{~mol} \%$ and the reactions were run for $18 \mathrm{~h}$. Next, these parameters were subjected to optimization employing the best performing catalysts, 7e and $\mathbf{7 f}$ (Table 3).

Table 3. Optimization of catalysts loading and the reaction time. ${ }^{\mathrm{a}}$

\begin{tabular}{llllll}
\hline entry & catalyst 7, $\mathrm{R}$ & $\begin{array}{l}\text { cat. } \\
\text { loading } \\
(\text { mol.\%) }\end{array}$ & $\begin{array}{l}\text { reaction } \\
\text { time }(\mathrm{h})\end{array}$ & $\begin{array}{l}\text { conve } \\
\text { rsion } \\
(\%)^{b}\end{array}$ & $\begin{array}{l}\text { ee } \\
(\%)^{c}\end{array}$ \\
\hline 1 & $\mathbf{7 e}, 3,5-\left(\mathrm{CF}_{3}\right)_{2} \mathrm{C}_{6} \mathrm{H}_{3}$ & 10 & 0.25 & $99^{c}$ & 91 \\
2 & $\mathbf{7 e}, 3,5-\left(\mathrm{CF}_{3}\right)_{2} \mathrm{C}_{6} \mathrm{H}_{3}$ & 5 & 0.25 & $99^{c}$ & 96 \\
3 & $\mathbf{7 e}, 3,5-\left(\mathrm{CF}_{3}\right)_{2} \mathrm{C}_{6} \mathrm{H}_{3}$ & 2 & 0.5 & 98 & \\
4 & $\mathbf{7 e}, 3,5-\left(\mathrm{CF}_{3}\right)_{2} \mathrm{C}_{6} \mathrm{H}_{3}$ & 2 & 1 & $99^{c}$ & 97 \\
5 & $\mathbf{7 e}, 3,5-\left(\mathrm{CF}_{3}\right)_{2} \mathrm{C}_{6} \mathrm{H}_{3}$ & 1 & 2 & 66 & \\
6 & $\mathbf{7 e}, 3,5-\left(\mathrm{CF}_{3}\right)_{2} \mathrm{C}_{6} \mathrm{H}_{3}$ & 1 & 3 & 93 & \\
7 & $\mathbf{7 e}, 3,5-\left(\mathrm{CF}_{3}\right)_{2} \mathrm{C}_{6} \mathrm{H}_{3}$ & 1 & 3.5 & $99^{c}$ & 98 \\
8 & $\mathbf{7 f}, 4-\mathrm{ClC}_{6} \mathrm{H}_{4}$ & 10 & 0.5 & $99^{c}$ & 98 \\
9 & $\mathbf{7 f}, 4-\mathrm{ClC}_{6} \mathrm{H}_{4}$ & 5 & 0.5 & 90 & \\
10 & $\mathbf{7 f}, 4-\mathrm{ClC}_{6} \mathrm{H}_{4}$ & 5 & 1 & $99^{c}$ & 98 \\
11 & $\mathbf{7 f}, 4-\mathrm{ClC}_{6} \mathrm{H}_{4}$ & 2 & 3 & 69 & \\
12 & $\mathbf{7 f}, 4-\mathrm{ClC}_{6} \mathrm{H}_{4}$ & 2 & 8 & $99^{c}$ & 97 \\
13 & $\mathbf{7 f}, 4-\mathrm{ClC}_{6} \mathrm{H}_{4}$ & 1 & 3 & 35 & \\
14 & $\mathbf{7 f}, 4-\mathrm{ClC}_{6} \mathrm{H}_{4}$ & 1 & 15 & 58 & 94 \\
\hline
\end{tabular}

a) The reactions were carried out at $-60{ }^{\circ} \mathrm{C}$ under argon. b) Conversion was calculated from GC data. ${ }^{\mathrm{c})}$ Determined by GC on a chiral column.

For catalyst 7e, with a loading of 10 or $5 \mathrm{~mol} \%$ the reaction was complete in under $15 \mathrm{~min}$ (entries 1 , 2). With $2 \mathrm{~mol} \%$, it took $30 \mathrm{~min}$ to reach nearly full conversion, with the starting materials completely disappearing after $1 \mathrm{~h}$ (entries 3,4). Even with 1 $\mathrm{mol} \%$, the reaction finished in under $4 \mathrm{~h}$ (entries 5-7). It is important to note that enantioselectivity remained high at low catalyst loadings (entries 4,7 ) Bipyridine $N, N$ '-dioxide $7 \mathbf{f}$ exhibited slightly reduced reactivity compared to $7 \mathrm{e}$. While with 10 and $5 \mathrm{~mol} \%$ it took under $1 \mathrm{~h}$ to achieve the complete conversion (entries 8-10), going down to $2 \mathrm{~mol} \%$ required $8 \mathrm{~h}$, whereas with $1 \mathrm{~mol} \%$ the full conversion was not reached even after $15 \mathrm{~h}$ (entries 11-14). However, enantioselectivity stayed high irrespective of the catalysts loading (entries 8, 10, 12, 14).

The optimization studies identified $7 \mathbf{e}$ as the catalyst of choice, outperforming other $N, N$ '-dioxide in terms of the reactivity at low catalyst loadings and excellent enantiocontrol. Therefore, it was taken further for investigating the reaction scope. The results are presented in Table 4.

With benzaldehyde 1a, the reaction worked well both on a small $(0.5 \mathrm{mmol})$ and a larger $(9.4 \mathrm{mmol})$ scale. For electron deficient aldehydes bearing nitro or trifluoromethyl groups (1b, 1c and 1d) enantioselectivity slightly dropped. Intriguingly, with $o$-nitrobenzaldehyde the product with the opposite absolute configuration was formed. In contrast, for aldehydes with donor groups (1e-1h,) the selectivity 
remained excellent. Halide substituents, irrespective of their nature or position around the ring (1i-1o), had no effect on the enantioselectivity which stayed high.

Table 4. Scope of asymmetric propargylation. ${ }^{\mathrm{a}}$

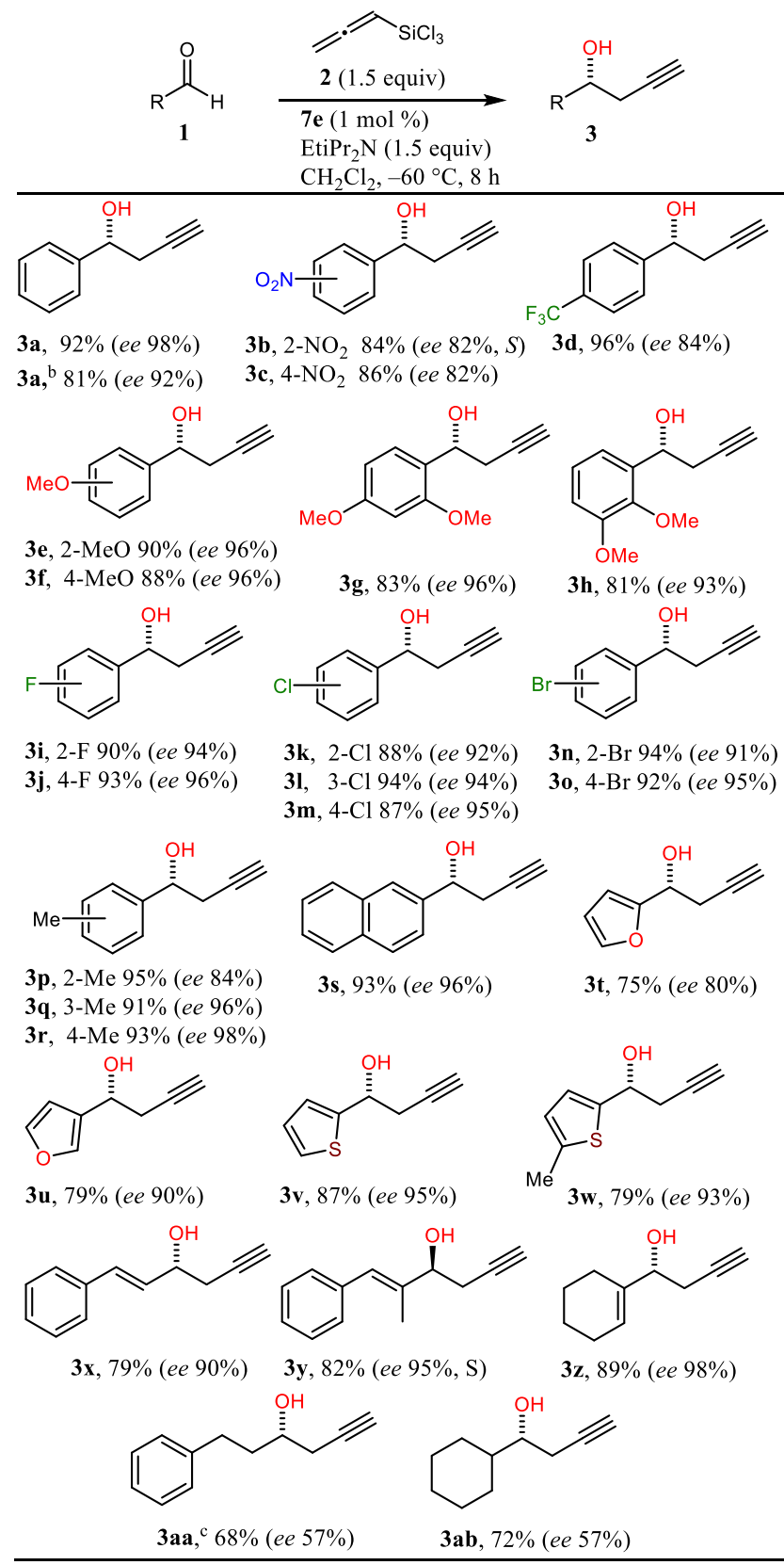

a) The reactions were carried out on a $0.5 \mathrm{mmol}$ scale under argon at $-60{ }^{\circ} \mathrm{C}$ for $8 \mathrm{~h}$ with $1 \mathrm{~mol} \%$ of catalyst $7 \mathbf{e}$, unless stated otherwise. The absolute configuration of products 3 was $R$ as assigned on the basis of literature data, unless stated otherwise. The yield is shown for isolated yield. Enantiopurity (shown in parenthesis) was determined by chiral GC or chiral HPLC, see SI for details. b) The reaction was performed on a $1.0 \mathrm{~g}(9.4 \mathrm{mmol}) \mathrm{scale} .{ }^{\mathrm{c})}$ The reaction was carried out with catalyst $7 \mathrm{~g}(10 \mathrm{~mol} \%)$ in $\mathrm{EtCN}$ at $-60^{\circ} \mathrm{C}$ for $18 \mathrm{~h}$.

For tolualdehydes 1p-1r, the position of the substituent mattered, with the $o$-substituent affecting the enantiocontrol the most. This is possibly due to increased steric hindrance in the proximity of the reaction site. 2-Naphthaldehyde 1 s mirrored the reactivity of benzaldehyde $\mathbf{1 a}$.

The set of electron-rich heteroaromatic aldehydes 1u-1w generally exhibited high enantioselectivities, except for furfural 1t, which gave alcohol 3t in only $80 \%$ ee. Cinnamaldehyde $\mathbf{1 x}$ and 1-cyclohexen-1carboxaldehyde $\mathbf{1 z}$ reacted uneventfully, whereas $\alpha$ methylcinnamaldehyde $\mathbf{1 y}$ gave the product $\mathbf{3 y}$ in high ee but with the opposite absolute configuration. Aliphatic aldehydes in Lewis-base catalyzed alkylations generally react slower with lower selectivity. The propargylation studied here was no exception: aldehydes $1 \mathbf{a a}$ and $\mathbf{1} \mathbf{a b}$ showed selectivity around $57 \%$ ee ( $c f$. the respective $\alpha, \beta$-unsaturated analogues 3x and 3z). Notably, 3-phenylpropanal 1aa with catalyst $7 \mathrm{e}$ in $\mathrm{CH}_{2} \mathrm{Cl}_{2}$ gave only $38 \%$ ee in $32 \%$ yield; the best result here was obtained with catalyst $7 \mathrm{~g}$ in $\mathrm{EtCN}$.

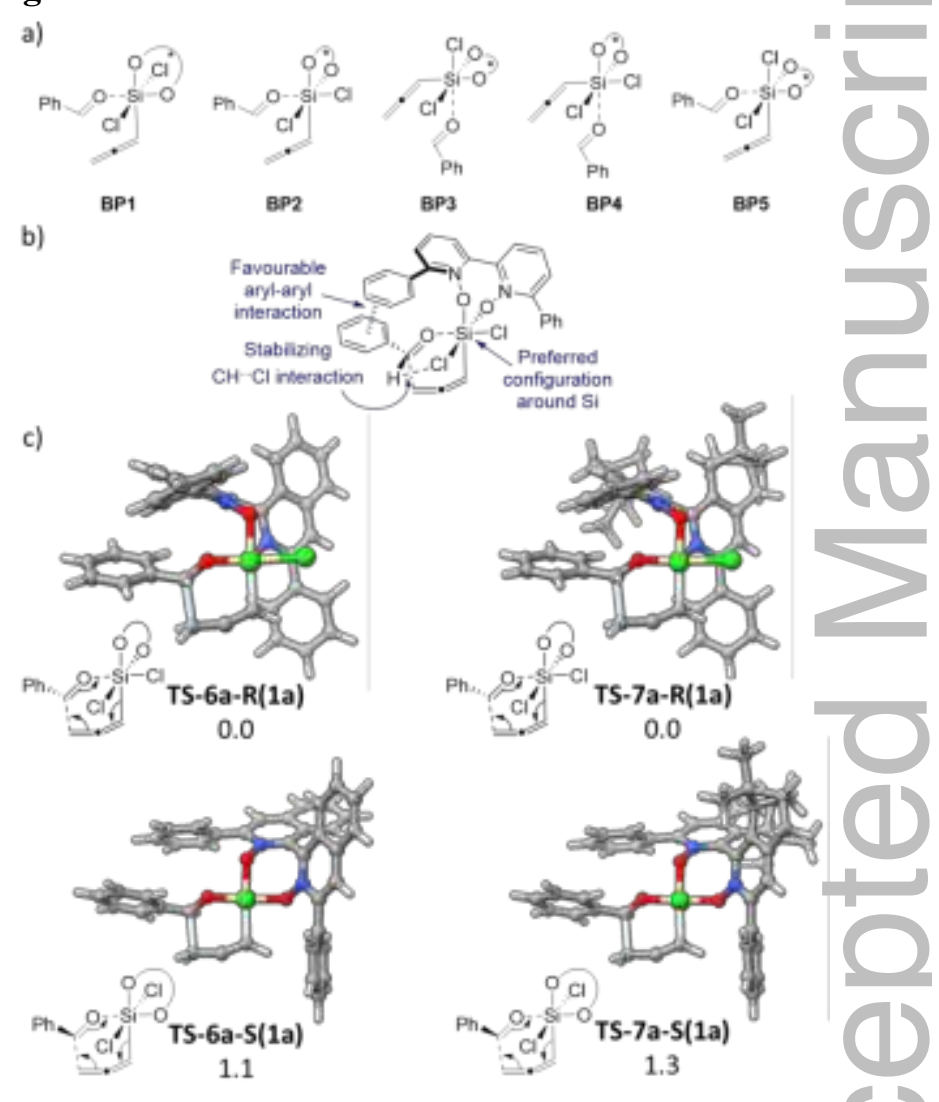

Figure 1. a) Stereochemical model; b) Five possible configurations of the hexacoordinate silicon intermediate preceding the stereocontrolling TS; c) Lowest-lying TS structures for the propargylation of benzaldehyde (1a, catalyzed by $6 \mathbf{a}$ and $7 \mathbf{a}$, along with relative energies in kcal $\mathrm{mol}^{-1}$. Structures for $\mathbf{6 a}$ taken from $\operatorname{Ref}^{[14 b]}$.

We next turned to computations to compare the key TS structures for selected examples of catalyst 7 with the previously studied catalysts 6 . Wheeler et al. previously showed ${ }^{[13]}$ that the stereocontrolling C-C bond forming step in these reactions can proceed via five distinct configurations around the silicon (BP1-5, 

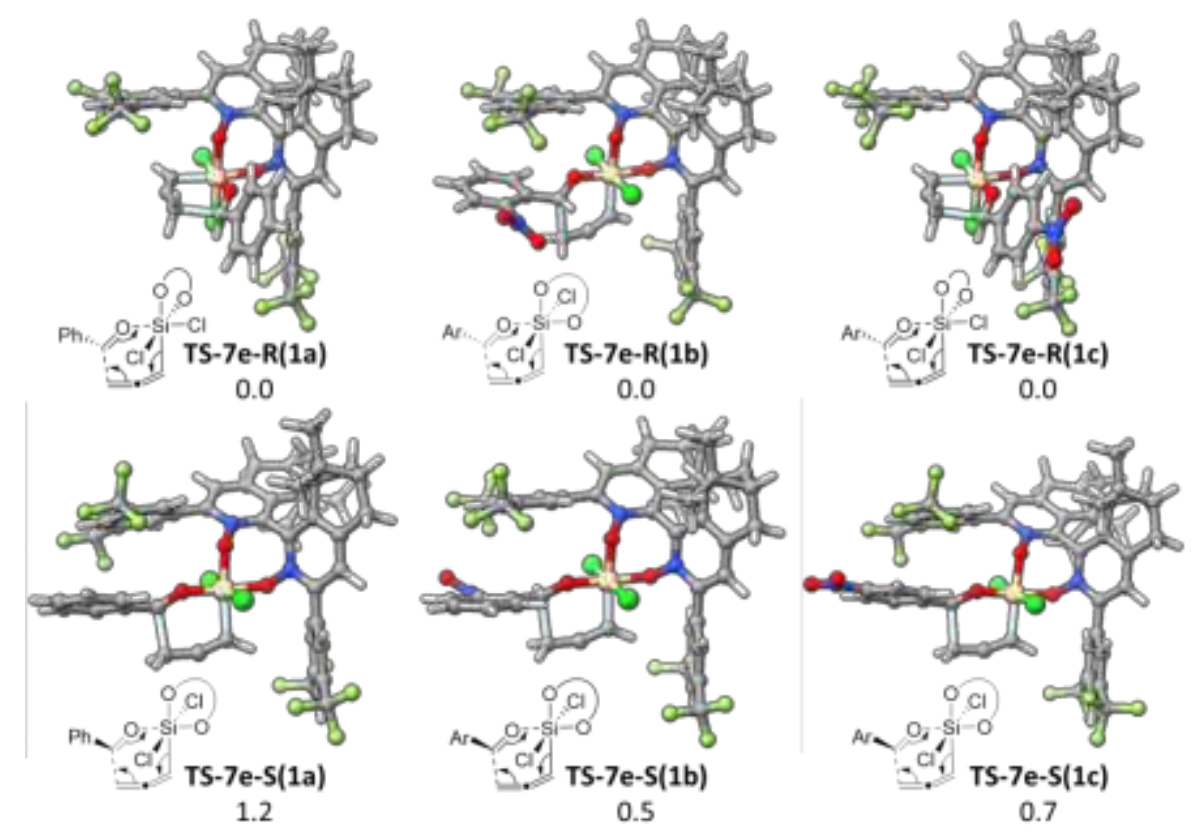

Figure 2. a) Lowest-lying TS structures for the propargylation of aldehydes $\mathbf{1 a}, \mathbf{1 b}$, and $\mathbf{1 c}$ catalyzed by $\mathbf{7 e}$, along with relative energies in $\mathrm{kcal} \mathrm{mol}^{-1}$.

see Figure 1a), with the selectivity primarily determined by the relative energy of the lowest-lying TS structures leading to the two possible stereoisomers. Studies of a model catalyst $\left(2,2^{\prime}-\right.$ bipyridine- $N, N^{\prime}$-dioxide) revealed that configuration BP2, with the chlorines in a cis arrangement and the nucleophile trans to one of the $\mathrm{N}$-oxides, is energetically favoured by more than $1.5 \mathrm{kcal} \mathrm{mol}^{-1}$ over the configuration with a trans-Cl arrangement (BP1) ${ }^{[14 b]}$ Consequently, for typical bipyridine- $N, N^{\prime}-$ dioxide derived catalysts the favourable TS structures exhibit configuration BP2. ${ }^{[14 a]}$ In these cases, the selectivity stems from the presence of favourable electrostatic interactions between the formyl $\mathrm{CH}$ and the nearby $\mathrm{Cl}$ in the TS structure leading to the $(R)$ alcohol (see Figure 1b). For catalysts bearing $\mathrm{Ph}$ substituents at the 3,3'-positions, however, Doney et $a l .{ }^{[14 b]}$ showed that stacking and $\mathrm{CH} / \pi$ interactions preferentially stabilize the TS structure derived from configuration BP1 leading to the $(S)$-product, resulting in different configurations around the Si for the most favourable TS structures leading to each stereoisomer. The selectivity then hinges on both the inherent stability of BP2, relative to BP1, combined with the net effect of non-covalent interactions in the corresponding TS structures.

The primary stereocontrolling TS structures from Ref. $^{[14 b]}$ for 6 a are depicted in Figure 1c, with TS $(R)$ lying $1.1 \mathrm{kcal} \mathrm{mol}^{-1}$ lower in energy than $\operatorname{TS}(S)$. As with other catalysts bearing $\mathrm{Ph}$ substituents at the 3,3'-positions, the lowest-lying TS structure leading to the $(R)$-alcohol (TS-6a-R) exhibits configuration BP2 while the most favourable TS leading to the $(S)$ product (TS-6a-S) follows BP5. The selectivity can then be attributed to the combination of the inherent stability of BP2 over BP1, combined with the balancing of stacking and $\mathrm{CH} / \pi$ interactions in both low-lying TS structures (see Figure 1b).

We used the latest version of $\mathrm{AARON}^{[18]}$ to automatically compute all low-lying TS structures fo. the propargylation of benzaldehyde (1a) catalyzed by 7a, 7e, and 7f at the PCM-B97-D/def2TZVP level o theory (See SI for more details). In all three cases, the lowest-lying TS structures are nearly identical to those previously computed for 6a. For example, the stereocontrolling TS structures for $7 \mathbf{a}$ are shown in Figure 1c. Furthermore, the energy difference between the stereocontrolling TS structures for $7 \mathbf{a}, 7 \mathbf{7 e}$, and $7 \mathbf{f}$ are similar to that computed for $\mathbf{6 a}$ (1.1 to 1.3

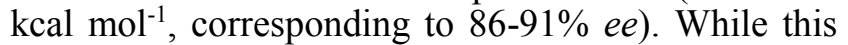
is not in exact quantitative agreement with the experimental data, it is consistent with the observed selectivities. Thus, while catalyst $\mathbf{7 a}$, for example, is not identical to the original catalyst design from Ref. ${ }^{[14 b]}$ (i.e. 6a), it behaves very similarly and achieves selectivity through the same mode identified previously (See Figure 1b). ${ }^{[14 b]}$ Thus, the experimental data presented for $7 \mathbf{a}$ provides at least indirect verification of the previous computationa predictions for $\mathbf{6 a}^{\left[{ }^{[14 b]}\right.}$

We also considered the propargylations of aldehydes $\mathbf{1 b}$ and $\mathbf{1 c}$ catalyzed by $7 \mathbf{e}$ (i.e. entries 3 and 4 from Table 4) to understand the impact of nitro substituents on the selectivity of this reaction. The key TS structures for aldehydes $\mathbf{1 a}, \mathbf{1 b}$, and $\mathbf{1 c}$ are shown in Figure 2. First, the lowest-lying $(R)$ and $(S)$-TS structures for $\mathbf{1 a}$ and $\mathbf{1 c}$ are nearly identical to those pictured in Figure 1c for 7a. For benzaldehyde (1a), the energy difference between the key stereocontrolling TS structures, TS-7e-R(1a) and TS- 
7e-S(1a), is $1.2 \mathrm{kcal} \mathrm{mol}^{-1}$. For $p$-nitrobenzaldehyde (1c), this energy difference is reduced to $0.7 \mathrm{kcal} \mathrm{mol}^{-}$ 1 , consistent with the experimental observation of reduced selectivity (i.e. entry 3 vs entry 1 in Table 4). Examining the structures in Figure 2, the reduced selectivity in the latter case can be explained by the modulation of the $\pi$-stacking interaction between the aromatic aldehyde and one of the bis- $\mathrm{CF}_{3}$-phenyl groups of the catalyst in the (S)-TS structure. For aldehyde 1c, the presence of a $p$-nitro group enhances the stacking interaction in the (S)-TS structure, due to the well-established substituent effects in $\pi$-stacking interactions, ${ }^{[19]}$ leading to stabilization of the disfavored TS and reduced selectivity.

In the case of $o$-nitrobenzaldehyde (1b), while computations indicate a further lowering of the energy of the $(S)$-TS compared to that for $(R)$, these data are not consistent with the experimental observation of reversed stereoselectivity. However, the results do show a qualitative change in the structure of the lowest-lying TS leading to the $(R)$ alcohol along with major changes in the energetic ordering of the possible TS structures for this reaction. More precisely, while the lowest-lying (S)-TS structure, TS-7e-S(1b), is similar to that found for 1a and 1c, the most favorable TS structure leading to the $(R)$-alcohol, TS-7e-R(1b), instead exhibits the same trans-chlorine configuration as TS-7e-S(1b). The structures for 1c analogous to those from 1a and 1c (not shown) is $0.6 \mathrm{kcal} \mathrm{mol}^{-1}$ higher in energy than TS-7e-R(1b). Overall, these results suggest that the stereoreversal in the case of aldehyde $\mathbf{1 b}$ can be attributed to the elimination of the favoured cischlorine pathway leading to the $(R)$-alcohol that is accessible for the other aldehydes.

\section{Conclusion}

Guided by previously reported computational predictions, ${ }^{[14 b]}$ a set of atropisomeric bipyridine $N, N$ '-dioxides $7 \mathbf{a}-7 \mathbf{j}$ were synthesized and tested as Lewis base catalysts for the asymmetric propargylation of aldehydes with trichloroallenylsilane. The catalysts are easy to prepare in four simple steps starting from methyl ketones readily available from commercial sources. Aryl-substituted derivatives proved to be highly active and showed high level of enantiocontrol; catalyst $7 \mathrm{e}$ was identified as the most efficient, retaining high reactivity even at $1 \mathrm{~mol} \%$ loading. Computations confirm that the key stereodetermining TS structures for this and other highly active and selective catalysts are nearly identical in most cases to those previously reported for catalyst $\mathbf{6 a} a^{[14 b]}$ The reaction scope includes a wide range of aromatic, heteroaromatic and unsaturated aldehydes, whereas aliphatic aldehydes proved to be challenging substrates.

\section{Experimental Section}

\section{General procedure for asymmetric propargylation}

The solution of allenyltrichlorosilane $(0.71 \mathrm{mmol}, 1.5$ equiv) in $0.5 \mathrm{~mL}$ DCM was added to a solution of catalyst $(0.0047 \mathrm{mmol}, 1 \mathrm{~mol} \%)$, diisopropylethylamine $(0.71$ mmol, 1.5 equiv) and aldehyde $(0.47 \mathrm{mmol})$ in DCM $(1.5$ $\mathrm{mL}$ ) under argon at $-60^{\circ} \mathrm{C}$. The mixture was stirred at the same temperature for 8 hours and then quenched with saturated $\mathrm{NH}_{4} \mathrm{Cl}$ solution $(2 \mathrm{~mL})$. The aqueous layer was extracted with $\mathrm{Et}_{2} \mathrm{O}(3 \times 20 \mathrm{~mL})$ and the combined organic extracts were washed with brine and dried over $\mathrm{Na}_{2} \mathrm{SO}_{4}$. The solvent was removed in vacuo and the residue was purified by flash chromatography on silica gel with a petroleum ether-ethyl acetate mixture 9:1. The enantiopurity of the resulting alcohols was determined by GC or HPLC with a chiral sorbents. The absolute configurations were assigned by comparing optical rotation values to the literature.

\section{Acknowledgements}

This work was supported by Russian Science Foundation Grant 18-73-10156 (Chemical part) and National Science Foundation Grant CHE-1665407 (Computational part). YF thanks the Japanese Government and the Loughborough University for a studentship. Portions of this work were conducted using high performance computing resources provided by the Georgia Advanced Computing Resource Center (http://gacrc.uga.edu). Molecular structure figures were generated using the SEQCROW plugin $^{[20]}$ for ChimeraX. ${ }^{[21]}$

\section{References}

[1] C. H. Ding, X. L. Hou, Chem. Rev. 2011, 111, 19141937.

[2] a) L. M. Geary, S. K. Woo, J. C. Leung, M. J. Krische, Angew. Chem. Int. Ed. 2012, 51, 2972-2976; b) K. D. Nguyen, D. Herkommer, M. J. Krische, J. Am. Chem. Soc. 2016, 138, 5238-5241; c) Y. Yang, I. B. Perry, G. Lu, P. Liu, S. L. Buchwald, Science 2016, 353, 144150 ; d) B. R. Ambler, S. K. Woo, M. J. Krische, ChemCatChem 2019, 11, 324-332.

[3] T. Thaima, F. Zamani, C. J. T. Hyland, S. G. Pyne, Synthesis 2017, 49, 1461-1480.

[4] a) M. Z. Wang, S. Khan, E. Miliordos, M. Chen, Org. Lett. 2018, 20, 3810-3814; b) A. S. Tsai, M. Chen, W. R. Roush, Org. Lett. 2013, 15, 1568-1571; c) H. Wang, P. Jain, J. C. Antilla, K. N. Houk, J. Org. Chem. 2013, 78, 1208-1215; d) M. Chen, W. R. Roush, J. Am. Chem. Soc. 2012, 134, 10947-10952; e) L. R. Reddy, Org. Lett. 2012, 14, 1142-1145; f) P. Jain, H. Wang, K. N. Houk, J. C. Antilla, Angew. Chem. Int. Ed. 2012, 51, 13911394; g) R. Haruta, M. Ishiguro, N. Ikeda, H. Yamamoto, J. Am. Chem. Soc. 1982, 104, 7667-7669; h) N. Ikeda, I. Arai, H. Yamamoto, J. Am. Chem. Soc. 1986, 108, 483-486; i) C. Q. Lai, J. A. Soderquist, Org. Lett. 2005, 7, 799-802.

[5] a) J. Zhao, S. J. T. Jonker, D. N. Meyer, G. Schulz, C. D. Tran, L. Eriksson, K. J. Szabo, Chem. Sci. 2018, 9, 
3305-3312; b) D. S. Barnett, S. E. Schaus, Org. Lett. 2011, 13, 4020-4023.

[6] a) J. A. Marshall, Chem. Rev. 1996, 96, 31-47; b) J. A. Marshall, J. Org. Chem. 2007, 72, 8153-8166; c) J. A. Marshall, N. D. Adams, J. Org. Chem. 1997, 62, 89768977; d) J. A. Marshall, K. Maxson, J. Org. Chem. 2000, 65, 630-633.

[7] a) S. E. Denmark, T. Wynn, J. Am. Chem. Soc. 2001, 123, 6199-6200; b) C. M. Yu, S. K. Yoon, K. Baek, J. Y. Lee, Angew. Chem. Int. Ed. 1998, 37, 2392-2395; c) C. M. Yu, S. K. Yoon, H. S. Choi, K. Baek, Chem. Commun. 1997, 763-764.

[8]a) S. E. Denmark, G. L. Beutner, Angew. Chem. Int. Ed. 2008, 47, 1560-1638; for the most recent reviews on application of heterocyclic $\mathrm{N}$-oxides as Lewis base catalysts, see: b) A. V. Malkov, Lewis-base Asymmetric Organocatalysis. In Sustainable Catalysis; RSC Green Chemistry; Royal Society of Chemistry: Cambridge, 2015, 317-350; c) P. Koukal, J. Ulč, D. Nečas, M. Kotora. Top. Heterocycl. Chem. 2017, 53, 29-58; d) Z. Wrzeszcz, B. Siedlecka, Molecules 2020, 25,330 .

[9] a) S. Kobayashi, K. Nishio, J. Am. Chem. Soc. 1995, 117, 6392-6393; b) U. Schneider, M. Sugiura, S. Kobayashi, Tetrahedron 2006, 62, 496-502.

[10] M. Nakajima, M. Saito, S. Hashimoto, Tetrahedron Asym. 2002, 13, 2449-2452.

[11] M. Nakajima, M. Saito, M. Shiro, S. Hashimoto, J. Am. Chem. Soc. 1998, 120, 6419-6420.

[12] J. S. Chen, B. Captain, N. Takenaka, Org. Lett. 2011, 13, 1654-1657.

[13] a) T. Lu, M. A. Porterfield, S. E. Wheeler, Org. Lett. 2012, 14, 5310-5313; b) T. X. Lu, R. X. Zhu, Y. An, S. E. Wheeler, J. Am. Chem. Soc. 2012, 134, 3095-3102; c) D. Sepulveda, T. X. Lu, S. E. Wheeler, Org. Biomol. Chem. 2014, 12, 8346-8353.

[14] a) B. J. Rooks, M. R. Haas, D. Sepulveda, T. X. Lu, S. E. Wheeler, ACS Catal. 2015, 5, 272-280; b) A. C. Doney, B. J. Rooks, T. X. Lu, S. E. Wheeler, $A C S$ Catal. 2016, 6, 7948-7955; for the synthesis of 6a, see: c) A. V. Malkov, M.-M Westwater, A. Gutnov, P. Ramírez-López, F. Friscourt, A. Kadlčíková, J. Hodačová, Z. Rankovic, M. Kotora, P. Kočovský, P. Tetrahedron 2008, 64, 11335-11348.

[15] Y. Fukazawa, V. Y. Vaganov, S. A. Shipilovskikh, A. E. Rubtsov, A. V. Malkov, Org. Lett. 2019, 21, 47984802.

[16] a) P. S. O'Hora, C. A. Incerti-Pradillos, M. A. Kabeshov, S. A. Shipilovskikh, A. E. Rubtsov, M. R. J. Elsegood, A. V. Malkov, Chem. Eur. J. 2015, 21, 45514555; b) C. A. Incerti-Pradillos, M. A. Kabeshov, P. S. O'Hora, S. A. Shipilovskikh, A. E. Rubtsov, V. A. Drobkova, S. Y. Balandina, A. V. Malkov, Chem. Eur. J. 2016, 22, 14390-14396.

[17] a) R. Hrdina, F. Opekar, J. Roithova, M. Kotora, Chem. Commun. 2009, 2314-2316; b) C. H. Geng, R. X. Zhu, D. J. Zhang, T. X. Lu, S. E. Wheeler, C. B. Liu, Mol Catal 2020, 483.

[18] Y. F. Guan, V. M. Ingman, B. J. Rooks, S. E. Wheeler, J. Chem. Theory Comput. 2018, 14, 52495261.

[19] S. E. Wheeler, J. Am. Chem. Soc. 2011, 133, 1026210274.

[20] https://github.com/QChASM/SEQCROW

[21] T. D. Goddard, C. C. Huang, E. C. Meng, E. F. Pettersen, G. S. Couch, J. H. Morris, T. E. Ferrir, Protein science : a publication of the Protein Society 2018, 27, 14-25. 


\section{FULL PAPER}

Optimization of Catalyst Structure for Asymmetric Propargylation of Aldehydes with

Allenyltrichlorosilane

Adv. Synth. Catal. Year, Volume, Page - Page

Vladimir Yu. Vaganov, Yasuaki Fukazawa,

Nikolay S. Kondratyev, Sergei A. Shipilovskikh, Steven E. Wheeler, Aleksandr E. Rubtsov, * and Andrei V. Malkov*

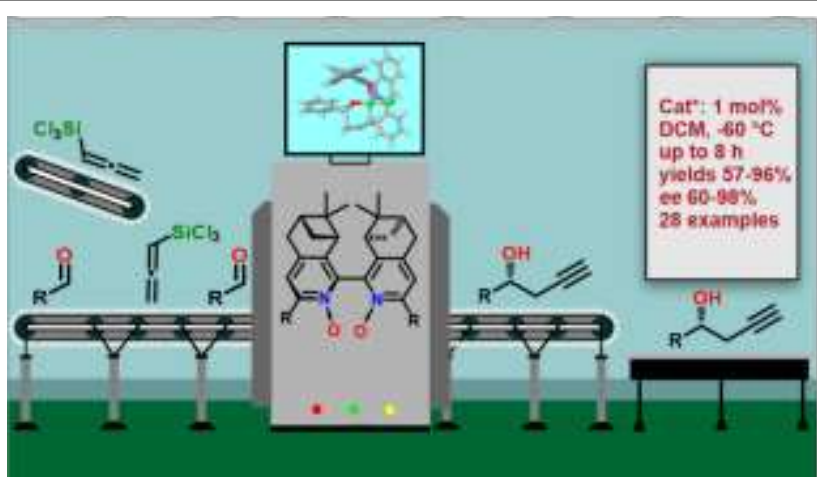

